\title{
LncRNA SLC16A1-AS1 Contributes to the Progression of Hepatocellular Carcinoma Cells by Modulating miR-411/MITD1 Axis
}

Chun Duan ( $\sim$ dc2021@yeah.net)

Wannan Medical College https://orcid.org/0000-0003-2419-7453

Bin Quan

Wannan Medical College

Ni Wang

Wannan Medical College

Jianghua Yang

Wannan Medical College

Yan-Lin Yu

Wannan Medical College

\section{Research Article}

Keywords: SLC16A1-AS1, miR-411, MITD1, hepatocellular carcinoma, tumorigenesis, metastasis

Posted Date: July 16th, 2021

DOI: https://doi.org/10.21203/rs.3.rs-685514/v1

License: (c) (i) This work is licensed under a Creative Commons Attribution 4.0 International License.

Read Full License

Version of Record: A version of this preprint was published at Journal of Clinical Laboratory Analysis on March 15th, 2022. See the published version at https://doi.org/10.1002/jcla.24344. 


\section{Abstract}

Background: Hepatocellular carcinoma (HCC) is a common malignancy with high morbidity. The current study aimed to explore the molecular mechannism of IncRNA SLC16A1-AS1 in the tumorigenesis of HCC.

Material and Methods: The expression of SLC16A1-AS1 and miR-411 were examined in clinical HCC tissues. HCC cell lines Hep3B and Huh-7 were employed and transfected with si-SLC16A1-AS1. The correlation between SLC16A1-AS1 and miR-411 was verified by luciferase reporter assay. Cell viability was detected by CCK-8 assay. Cell migration and invasion capacity were examined by transwell assay. The protein level of MITD1 was analyzed by western blotting.

Results: The expression of SLC16A1-AS1 markedly increased in HCC tissues and cell lines. Subsequent studies identified SLC16A1-AS1 as a downstream target of miR-411. In addition, SLC16A1-AS1 knockdown and miR-411 overexpression significantly stagnated progression of HCC cells. SLC16A1-AS1 knockdown also downregulated MITD1 levels.

Conclusion: Our findings showed that SLC16A1-AS1 was overexpressed in HCC cells and tissues. SLC16A1-AS1 promoted the malignant characteristics of HCC cells and acted as an oncogene. Its regulatory effect may be associated with miR-411/MITD1 axis. Therefore, SLC16A1-AS1 has a potential be used as a biomarker or therapeutic target for the treatment of HCC.

\section{Introduction}

Hepatocellular carcinoma (HCC) is the fifth most common cancer in the world, and male mortality ranks second, especially in Africa and Asia ${ }^{[1]}$. Although the treatment of $\mathrm{HCC}$ has made progress in recent decades, HCC remains the third leading cause of cancer-related deaths worldwide. In approximately $90 \%$ of cases, hepatocellular carcinoma is related to liver cirrhosis ${ }^{[2]}$. One third of patients with liver cirrhosis will develop HCC in the future ${ }^{[3]}$. Liver cirrhosis is an advanced scar formation process. Due to long-term liver injury, healthy liver tissue is replaced by nodules and scar tissue, and the scar tissue is surrounded by fiber bundles ${ }^{[4]}$. Irreversible liver cirrhosis may eventually develop into HCC. Therefore, finding new targets for therapeutic and diagnostic methods will help to the early diagnosis and intervention of HCC.

Studies have shown that less than $1.5 \%$ of human genome has a protein-coding function, and some of the rest contains functionally conserved non-coding series ${ }^{[5]}$. A large part of the genome is transcribed into non-coding RNAs (ncRNAs), with no or low protein coding functions. NcRNA can be divided into several families according to its molecular size ${ }^{[6]}$. LncRNA is defined as a transcript with a length of more than 200 nucleotides $^{[7]}$. Recently, a lot of attention has been focused on IncRNA. With the development of genome-wide transcriptomics analysis, IncRNA has been confirmed to participate in a variety of biological activities, such as cell cycle regulation, apoptosis, differentiation and other life activities $[8,9]$. The latest research shows that IncRNA can affect HCC metastasis-related molecules at the transcription 
level and post-transcription level, and provide potential diagnostic indicators and therapeutic targets for HCC patients ${ }^{[10]}$.

The current study aims to better understand the molecular mechanism of SLC16A1-AS1 in the occurrence and development of HCC. The expression level of SLC16A1-AS1 in clinical specimens and cells was determined. Function assay uncovered the role of SLC16A1-AS1 in the malignant phenotypes of HCC cells. Further study investigated the molecular interaction between SLC16A1-AS1 and miR-411/MITD1 axis. This article analyzes in detail the role of SLC16A1-AS1 in the progression of HCC, which will help to understand the pathogenesis of HCC.

\section{Materials And Methods}

Clinical specimens

The present study was conducted with the permission of the Ethics Committee of Wannan Medical College. All participants provided written informed consent. HCC tissues were surgically resected and stored in liquid nitrogen for further analysis.

Cell culture and reagents

The human HCC cell lines (Hep3B and Huh-7) and human normal hepatic cell line (LO2) were purchased from ATCC ((American Type Culture Collection, Manassas, US). The cells were cultured in DMEM medium supplemented with $10 \%$ fetal bovine serum (GIBCO, MA, USA). SiRNA-SLC16A1-AS1 and negative control miRNA were synthesized by Biossci Company (Wuhan, China). Transfections were performed using Lipofectamine 2000 (Invitrogen, CA, USA).

Quantitative real-time PCR (qRT-PCR)

The total RNA was isolated from cells and tissues using Trizol reagent (Life Technologies, USA). Firststrand cDNA was carried out using using reverse transcription Reagents (ABI, CA), QRT-PCR was performed using SYBRH Select Master Mix for CFX (Invitrogen) and using the CFX Connet TM real-time PCR system (Bio-Rad, USA). GAPDH and U6 were chosen as the internal standard.

Cell viability assay

Cell viability was measured using Cell Counting Kit-8 assay (Beyotime, China). After being inoculated into 96-well plates at a density of $2 \times 10^{3}$ cells/well, cells were stained with $20 \mu \mathrm{L}$ of CCK8 reagent 48 hours after transfection. Cell viability was determined at the $450 \mathrm{~nm}$ absorbance.

Invasion assay

In invasion test, $1 * 10^{5}$ cells were inoculated into the upper chambers ( $8 \mu \mathrm{m}$ pore diameter, BD, USA) with serum-free medium. Complete medium $(600 \mu \mathrm{L})$ was added to the bottom chamber to simulate the 
chemoattractant. $48 \mathrm{~h}$ after incubation, cells on the top membrane were wiped out. Invaded cells on the bottom surface were fixed and stained. counted in five random scopes and photographed.

Wound healing assay

Cells were seeded in 6-well plates. After $12 \mathrm{~h}$ of incubation, wounds were generated with a $200 \mu \mathrm{L}$ pipette tip. Cells were washed and incubated with serum-free medium for $48 \mathrm{~h}$. The wounded gap was photographed at different time points. Cell migration was caculated as wound closure $(\%)=($ wounded area $(\mathrm{A} 0-\mathrm{A} 1) /$ wounded area $\mathrm{A} 0) \times 100 \%$.

Dual-luciferase assay

The psi-CHECK-2 psi-check Luciferase Expression Reporter (Promega) vector was used to synthesize SLC16A1-AS1 vector. Hep3B and Huh-7 cells were used for the dual-luciferase-reporter assays. Luciferase activity was measured and compared $48 \mathrm{~h}$ later. Luciferase assay was performed $48 \mathrm{~h}$ after transfection using a Dual-Luciferase Reporter Assay System (Promega, WI, USA).

Western blot

Cells were washed twice with PBS, and total protein was extracted using RIPA buffer (Beyotime Biotechnology, China). Protein concentration was determined using a BCA Protein Assay Kit (Beyond Biotechnology). Protein amples were separated using 10 \% SDS-polyacrylamide gel (Solarbio, Beijing) after denaturation. Then, proteins were transferred to a PVDF membrane (Millipore, USA) which were blocked with $5 \%$ skimmed milk for $1 \mathrm{~h}$. The blots were incubated overnight with the first antibodies (anti MITD1 antibody, 1:5000, Santa Cruz; anti-GAPDH antibody, 1:5000, Santa Cruz.). ECL (Millipore) After incubation with secondary antibodies, an enhanced chemiluminescence reagent was applied for detection.

Statistical analysis

All statistical analyses used SPSS v22.0 (SPSS Inc., Chicago, USA). Each experiment was repeated at least three times. Data was shown as mean \pm standard deviation. The significance of differences between groups were assessed by Student's $t$ test and ANOVA test. P-values $<0.05$ were considered statistically significant.

\section{Results}

SLC16A1-AS1 is up-regulated in HCC cells and tissues

To determine the expression of SLC16A1-AS1 in HCC tissues, we explored the expression of SLC16A1AS1 in the TCGA data portal of starbasever3.0. As shown in Fig. 1A, compared with normal tissues, SLC16A1-AS1 increased significantly in HCC tissues. Accordingly, the expression of SLC16A1-AS1 in HCC tissues and cells was significantly higher than that in normal cells (Fig. 1C and D). Kaplan-Meier analysis 
was performed, and the data revealed that compared with patients with low SLC16A1-AS1, patients with high SLC16A1-AS1 had a worse overall survival time (Fig. 1B). In addition, the subcellular distribution experiment was used to study the expression area of SLC16A1-AS1 in HCC cells. The results showed that SLC16A1-AS1 was mainly expressed in the nucleus rather than the cytoplasm (Fig. 1E and F). In summary, our findings suggest that SLC16A1-AS1 is overexpressed in HCC.

SLC16A1-AS1 regulates proliferation and invasion of HCC in vitro

Considering the increased expression of SLC16A1-AS1 in HCC, we explored the effect of SLC16A1-AS1 gene knockdown on the malignant phenotype of HCC cells. By transfection of siRNA, the expression level of SLC16A1-AS1 in Hep3B and Huh-7 cells was significantly reduced (Fig. 2A and B). Then CCK-8 analysis was performed, and the results showed that SLC16A1-AS1 knockdown significantly inhibited the proliferation of the two HCC cell lines (Fig. $2 \mathrm{C}$ and D). At the same time, the transwell invasion test was used to detect the invasion ability of the cells. The data showed that compared with the NC group, the number of invasive cells in the si-SLC16A1-AS1 group was significantly reduced (Fig. 2E). Cell migration was measured by wound healing experiments. The data showed that SLC16A1-AS1 silencing significantly inhibited cell migration (Fig. 2F and G). The above results suggest that SLC16A1-AS1 gene knockdown can inhibit the proliferation and invasion of HCC cells.

SLC16A1-AS1 acts as a ceRNA in HCC cells

It has been confirmed that IncRNA regulates the expression of miRNA through the sponge effect. Therefore, we used the TargetScan database to identify candidate miRNAs that interact with SLC16A1AS1. The potential binding site of miR-411 is shown in Fig. 3A. The expression of miR-411 was detected in the tissues, and the same expression was also obtained in the transitional cell carcinoma of the bladder (Fig. 3B). The expression level of miR-411 in HCC cells was also detected and found that its expression level was down-regulated as expected (Fig. 3C). To further verify our hypothesis, we performed a luciferase reporter analysis to explore the correlation between miR-411 and SLC16A1-AS1. The results showed that miR-411 significantly inhibited the luciferase activity of the cells transfected with SLC16A1AS1-Wt, while the luciferase activity of the SLC16A1-AS1-Mut group was not affected (Fig. 3D and 3E). As mentioned above, the above data indicate that SLC16A1-AS1 acts as a ceRNA to regulate the expression of miR-411 in HCC cells.

MiR-411 regulates malignant phenotypes of HCC cell

The TCGA data portal of starbasever3.0. As shown in Fig. 4A and B, compared with normal tissues, miR411 decreased and MITD1 increased significantly in HCC tissues. To study the biological functions of miR-411 in vitro. After transfection with miR-411 mimics, the expression of miR-411 in HCC cells was detected. The data showed that miR-411 was significantly increased in HCC cells (Fig. 4C and D). Cell viability assays showed that miR-411 overexpression remarkedly stagnated cell proliferation (Fig. 4E and F). Then the cell invasion experiment showed that in the two HCC cell lines, the number of invaded cells was obviously reduced after the miR-411 mimic was transfected compared with the miR-411 mimic NC 
(Fig. 4G). In summary, these findings suggest that miR-411 plays a tumor suppressor role in the proliferation and invasion of HCC cells.

MiR-411 directly targets MITD1

With the help of open access databases (TargetScan, MiRanda and Starbase3.0), we determined that MITD1 is a potential downstream target of miR-411. Therefore, a dual luciferase reporter plasmid containing WT or MITD1 3'UTR mutants was synthesized (Fig. 5A). The plasmid containing mitd13'UTR was co-transfected with miR-411 mimic or NC mimic into Hep3B and Huh-7 cells. Determination of luciferase activity. The data from the dual-luciferase reporter gene analysis showed that the 3'UTR mutation of MITD1 abolished the inhibitory ability of miR-411 compared to the wild type (Fig. 5B and C). In addition, miR-411 overexpression significantly reduced MITD1 protein levels (Fig. 5D and E). In conclusion, our results confirm that miR-411 directly regulates MITD1 expression.

\section{Discussion}

Numerous evidences showed that IncRNAs regulate a variety of biological processes and play an important role in tumorigenesis and progression. Recent studies have shown that dysregulated IncRNAs can be used as effective therapeutic targets for HCC. At present, some IncRNAs have been found to have carcinogenic effects. For example, IncRNA SUM01P3, SNHG9, XIST were found to be upregulated in HCC and acted as oncogene ${ }^{[11-13]}$. These findings indicated that IncRNAs have great potential to be used as therapeutic targets in HCC treatment. In our present study, we analyzed the expression of SLC16A1-AS1 in HCC tissues and cell lines. SLC16A1-AS1 was found to be markedly upregulated both in tissues and cells. Then, we conducted a functional study to explore the role of SLC16A1-AS1 in HCC cells. SLC16A1-AS1 silencing significantly suppressed cell proliferation, colony formation and invasion of HCC cells. Collectively, the results verified that SLC16A1-AS1 may act as an oncogene in HCC cells.

Previous studies have shown that IncRNAs may exert the ability to function as a miRNA sponge, competing for miRNA, and modulate the expression of miRNA. For instance, LncRNA XIST promotes HBV related HCC by upregulating TRIM25 ${ }^{[14]}$. LncRNA Lnc-APUE promotes tumor growth by regulating miR20b/E2F1 axis in HCC ${ }^{[15]}$. LncRNA SMASR inhibits lung cancer progression by negatively regulating TGF$\beta /$ Smad signaling ${ }^{[16]}$. These studies enlightened us to explore the potential miRNAs that interact with SLC16A1-AS1. Using open access database prediction, we chose miR-411 for further study. Further study confirmed the role of miR-411 in HCC and the regulatory relationship between SLC16A1-AS1 and miR411.

MITD1 encodes a protein that regulates the activity of ESCRT-III and is necessary for normal cytokinesis. MITD1 has been shown to be involved in the shedding phase of cytokinesis ${ }^{[17]}$. Disorders of shedding may lead to tumorigenesis and genetic instability, especially when combined with mitotic stress caused by oncogenes ${ }^{[18,19]}$. In our study, we identified MITD1 as a down-stream target of miR-411. SLC16A1-AS1 
regulated MITD1 expression by interacting with miR-411. These data suggested that SLC16A1-AS1 exerts its function through the miR-411/MITD1 axis.

\section{Conclusion}

In conclusion, our study shows that SLC16A1-AS1 is up-regulated in HCC tissues and cells. SLC16A1-AS1, as an oncogene in HCC, exerts a carcinogenic effect by regulating the miR-411/MITD1 axis. Our research helps to understand the role of SLC16A1-AS1 in HCC, suggesting that SLC16A1-AS1 may be used as a target for liver cancer treatment.

\section{Declarations}

\section{Ethics approval and consent to participate:}

The present study was approved by the Institutional Ethics Committee of Yijishan Hospital of Wannan Medical College and carried out according to the guideline of the ethical management. Prior written consent was well informed and signed by all participants.

\section{Consent for publication:}

All patients signed consent for publication.

\section{Availability of data and material:}

The datasets used and analysed during the current study are available from the corresponding author on reasonable request.

\section{Competing interests:}

All authors have no conflicts of interest or financial ties to disclose.

\section{Funding:}

Not available.

\section{Acknowledgements:}

Not available.

\section{Authors' contributions:}

Chun Duan contributed to the conception and design of the work. Bin Quan conducted the experiments. $\mathrm{Ni}$ Wang and Jianghua Yan analyzed data and wrote this manuscript.Yan-Lin Yu revised and reviewed the manuscript. All authors contributed to final approval of the version to be published and agree to be accountable for all aspects of the work. 


\section{References}

1. Siegel, R. L., Miller, K. D., Jemal, A. (2020) Cancer statistics, 2020. CA Cancer J Clin. 70, 7-30.

2. Burra, P., Zanetto, A., Germani, G. (2018) Liver Transplantation for Alcoholic Liver Disease and Hepatocellular Carcinoma. Cancers (Basel). 10.

3. Tangjarusritaratorn, T., Tangjittipokin, W., Kunavisarut, T. (2021) Incidence and Survival of Hepatocellular Carcinoma in Type 2 Diabetes Patients with Cirrhosis Who Were Treated with and without Metformin. Diabetes Metab Syndr Obes. 14, 1563-1574.

4. Yasser, M. B., Abdellatif, M., Emad, E., Jafer, A., Ahmed, S. et al. (2021) Circulatory miR-221 \& miR-542 expression profiles as potential molecular biomarkers in Hepatitis $C$ Virus mediated liver cirrhosis and hepatocellular carcinoma. Virus Res. 296, 198341.

5. Lander, E. S. (2011) Initial impact of the sequencing of the human genome. Nature. 470, 187-197.

6. Pea, A., Jamieson, N. B., Braconi, C. (2021) Biology and Clinical Application of Regulatory RNAs in Hepatocellular Carcinoma. Hepatology. 73 Suppl 1, 38-48.

7. St, L. G., Wahlestedt, C., Kapranov, P. (2015) The Landscape of long noncoding RNA classification. Trends Genet. 31, 239-251.

8. Huang, Z., Zhou, J. K., Peng, Y., He, W., Huang, C. (2020) The role of long noncoding RNAs in hepatocellular carcinoma. Mol Cancer. 19, 77.

9. Han, T. S., Hur, K., Cho, H. S., Ban, H. S. (2020) Epigenetic Associations between IncRNA/circRNA and miRNA in Hepatocellular Carcinoma. Cancers (Basel). 12.

10. Lv, E., Sheng, J., Yu, C., Rao, D., Huang, W. (2021) LncRNA influence sequential steps of hepatocellular carcinoma metastasis. Biomed Pharmacother. 136, 111224.

11. Hu, S., Liu, J., Feng, S., Wang, Y., Liu, H. (2021) LncRNA SUMO1P3 acts as a prognostic biomarker and promotes hepatocellular carcinoma growth and metastasis. Aging (Albany NY). 13.

12. Ye, S., Ni, Y. (2021) IncRNA SNHG9 Promotes Cell Proliferation, Migration, and Invasion in Human Hepatocellular Carcinoma Cells by Increasing GSTP1 Methylation, as Revealed by CRISPR-dCas9. Front Mol Biosci. 8, 649976.

13. Wang, J., Yin, G., Bian, H., Yang, J., Zhou, P. et al. (2021) LncRNA XIST upregulates TRIM25 via negatively regulating miR-192 in hepatitis B virus-related hepatocellular carcinoma. Mol Med. 27, 41.

14. Wang, J., Yin, G., Bian, H., Yang, J., Zhou, P. et al. (2021) LncRNA XIST upregulates TRIM25 via negatively regulating miR-192 in hepatitis B virus-related hepatocellular carcinoma. Mol Med. 27, 41.

15. Li, S. Y., Zhu, Y., Li, R. N., Huang, J. H., You, K. et al. (2021) LncRNA Lnc-APUE is Repressed by HNF4alpha and Promotes G1/S Phase Transition and Tumor Growth by Regulating MiR-20b/E2F1 Axis. Adv Sci (Weinh). 8, 2003094.

16. Xu, L., Liu, W., Li, T., Hu, Y., Wang, Y. et al. (2021) Long non-coding RNA SMASR inhibits the EMT by negatively regulating TGF-beta/Smad signaling pathway in lung cancer. Oncogene. 
17. Lee, S., Chang, J., Renvoise, B., Tipirneni, A., Yang, S. et al. (2012) MITD1 is recruited to midbodies by ESCRT-III and participates in cytokinesis. Mol Biol Cell. 23, 4347-4361.

18. Shen, H., Wang, Z., Ren, S., Wang, W., Duan, L. et al. (2020) Prognostic biomarker MITD1 and its correlation with immune infiltrates in hepatocellular carcinoma (HCC). Int Immunopharmacol. 81, 106222.

19. Sadler, J., Wenzel, D. M., Williams, L. K., Guindo-Martinez, M., Alam, S. L. et al. (2018) A cancerassociated polymorphism in ESCRT-III disrupts the abscission checkpoint and promotes genome instability. Proc Natl Acad Sci U S A. 115, E8900-E8908.

\section{Figures}


A

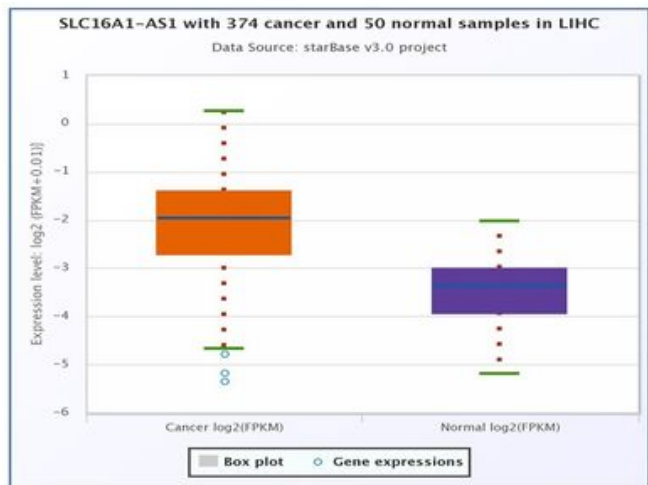

C

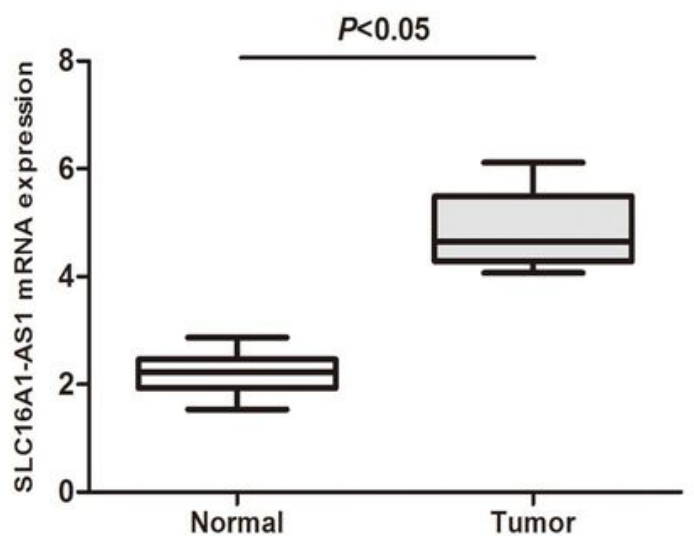

E

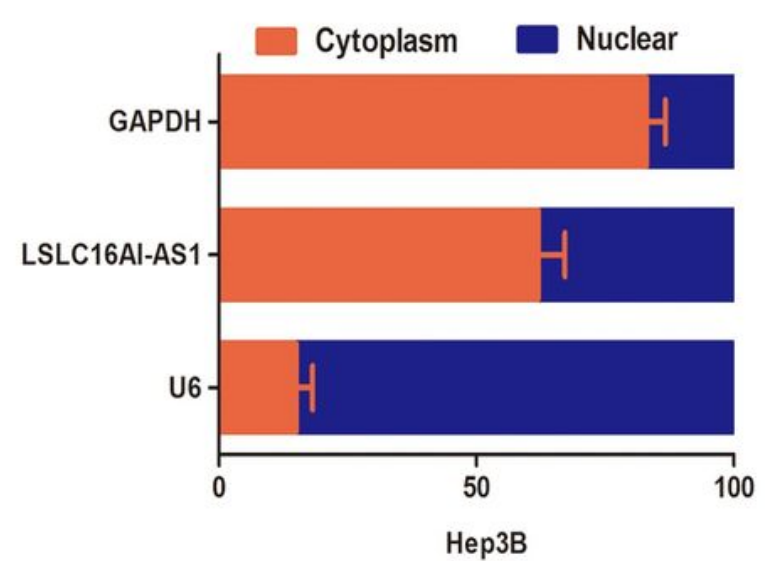

B

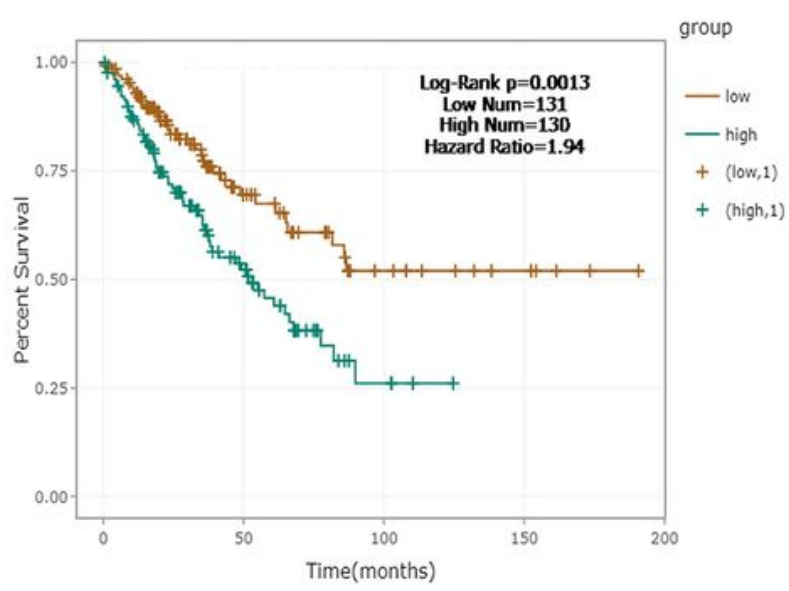

D

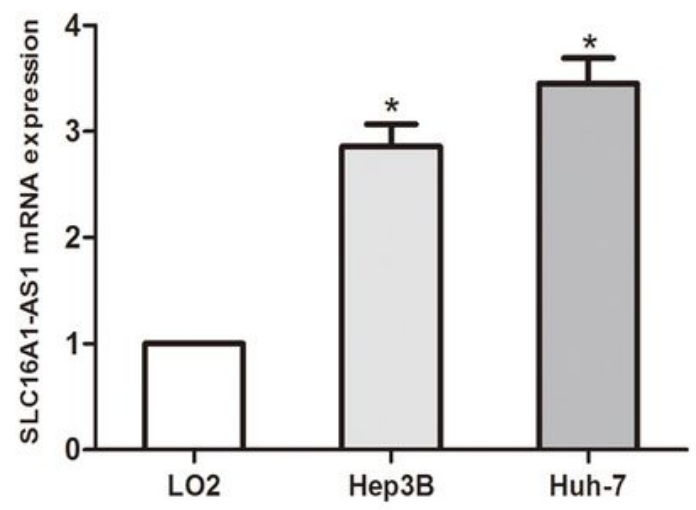

$\mathbf{F}$

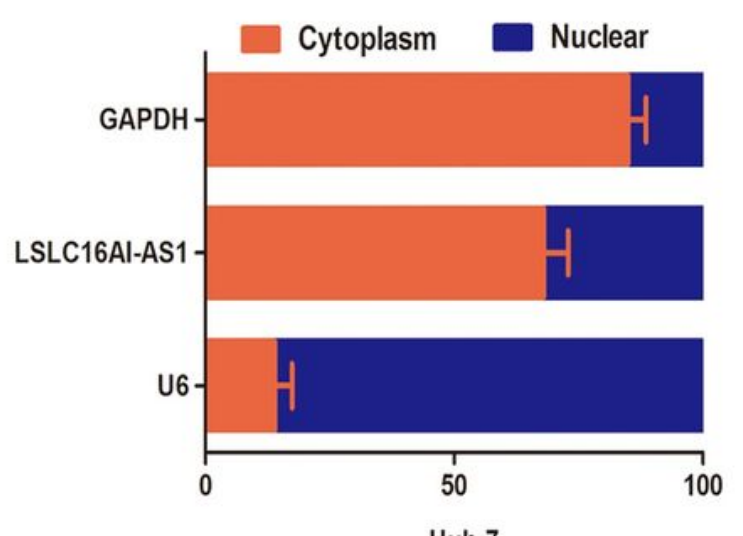

Huh-7

\section{Figure 1}

SLC16A1-AS1 is up-regulated in HCC cells and tissues. (A) Data from Starbase 3.0 shows the expression of LncRNA SLC16A1-AS1 in HCC tissues and normal tissues. (B) Survival analysis of SLC16A1-AS1 in patients with HCC. (C) LINC01287 expression in HCC tissues and normal tissues. (D) LINC01287 expression in HCC cells (Hep3B and Huh-7) and human normal hepatic cell line (LO2). (E and F) The 
distribution of LINC01287 in the subcellular fractions of Hep3B and Huh-7 cells. U6 and GAPDH were used as nuclear and cytoplasmic markers, respectively.
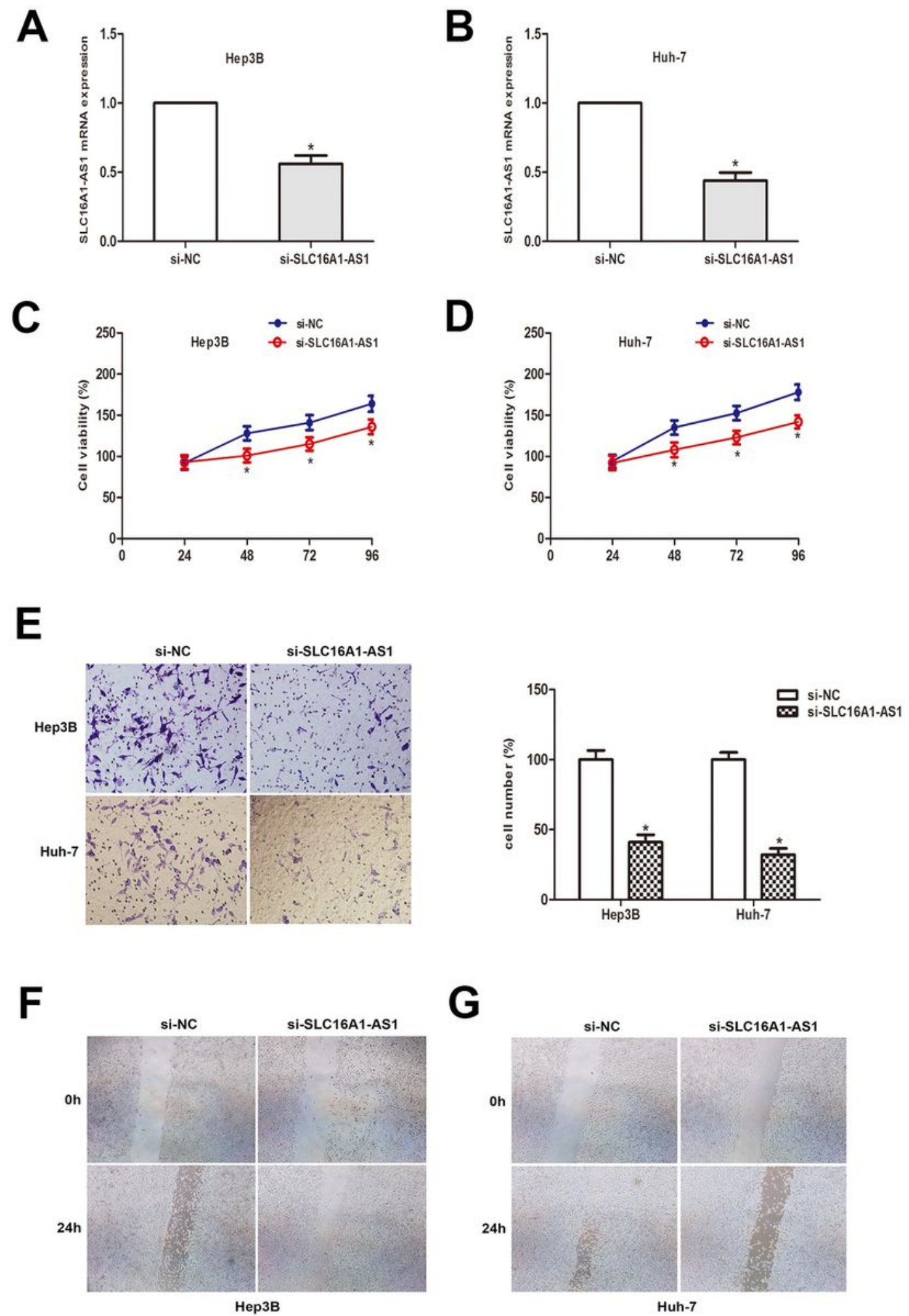

Figure 2

SLC16A1-AS1 regulates proliferation and invasion of HCC in vitro. (A and B) qRT-PCR results showed siSLC16A1-AS1 were transfected into Hep3B and Huh-7 cells successfully. (C and D) CCK-8 assay was performed to determine cell viability of cells transfected with si-LINC01287. (E) Transwell invasion assay 
was performed to investigate invasive capacity of cells transfected with si-LINC01287. (F and G) Wound healing assay was performed to evaluate the migratory level. $\left({ }^{*} \mathrm{P}<0.05\right)$
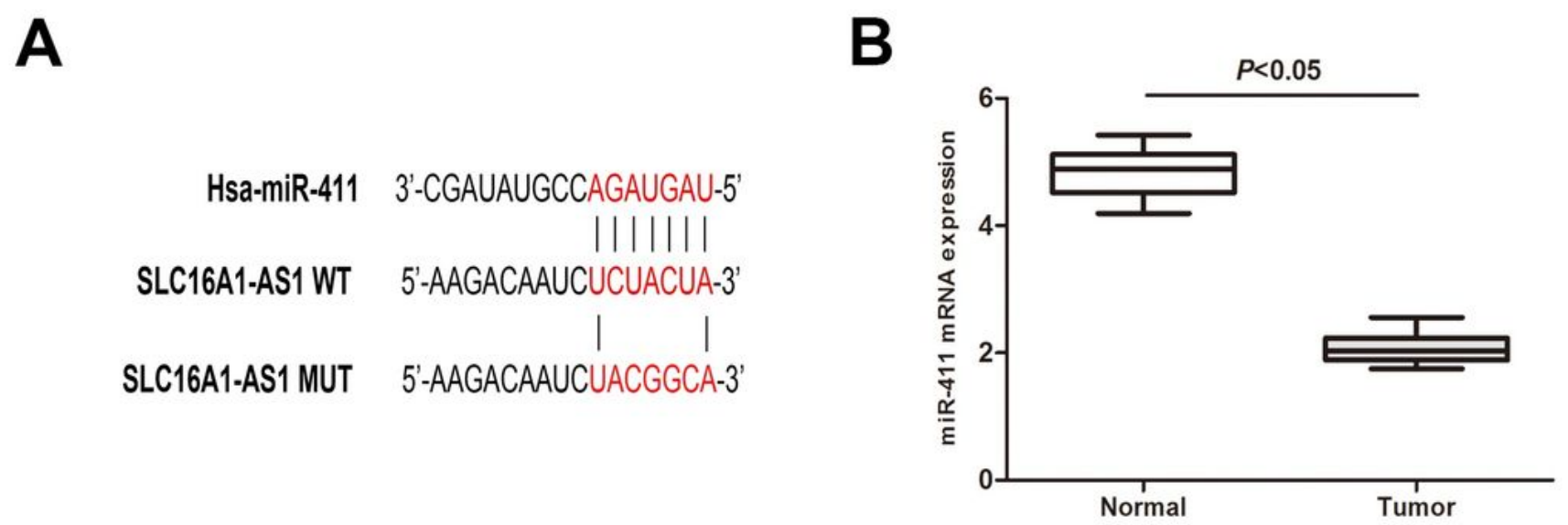

C

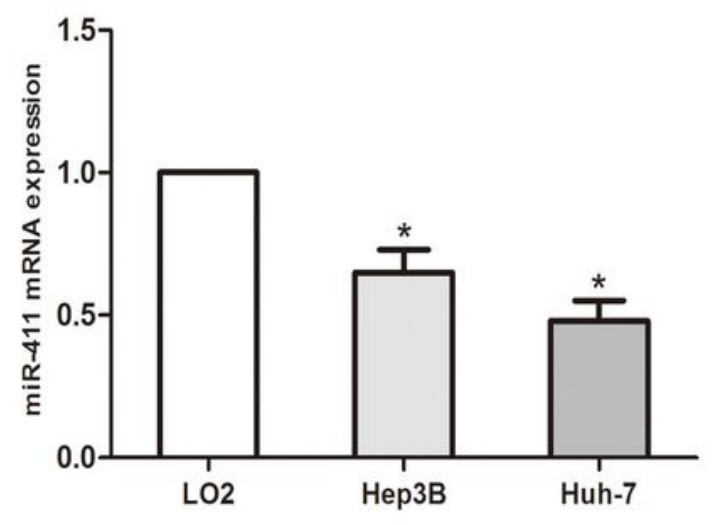

\section{D}

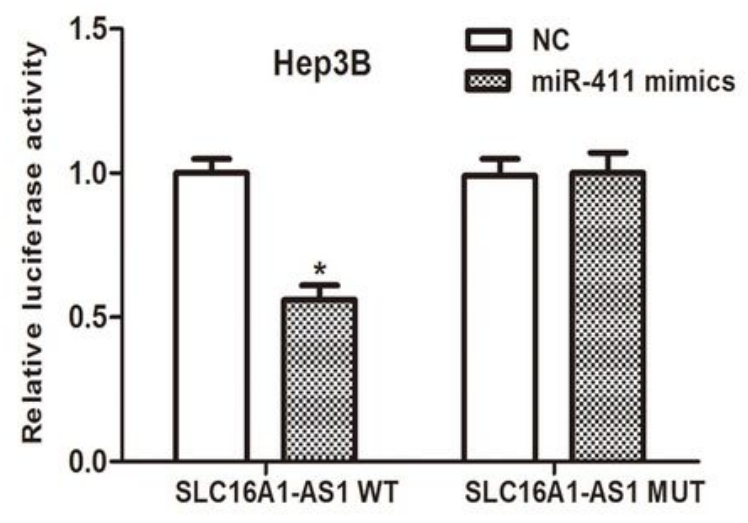

$\mathbf{E}$

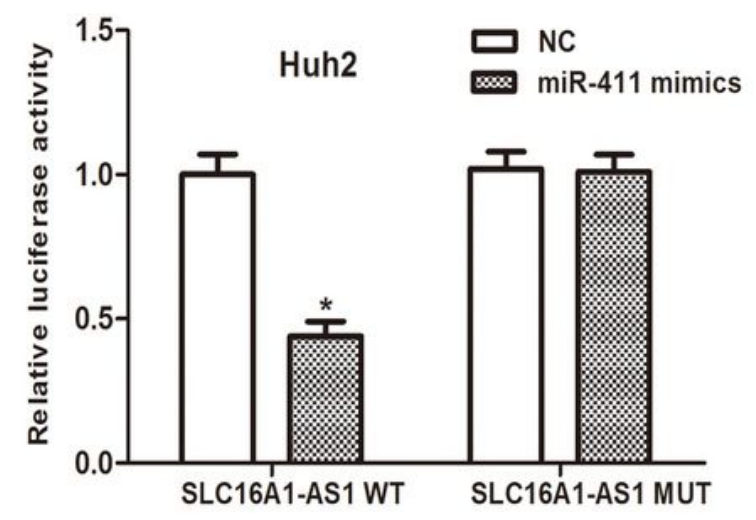

Figure 3

SLC16A1-AS1 acts as a ceRNA in HCC cells. (A) Putative binding site of miR-411 on LINC01287. (B) MiR411 expression in HCC tissues and normal adjacent tissues was measured using qRT-PCR. (C) MiR-411 expression in HCC cells was detected by qRT-PCR. (D and E) Dual-luciferase reporter assay was 
performed to determine luciferase activity in cells co-transfected with miR-411 mimics and SLC16A1-AS1WT or SLC16A1-AS1-MUT. (*P<0.05)
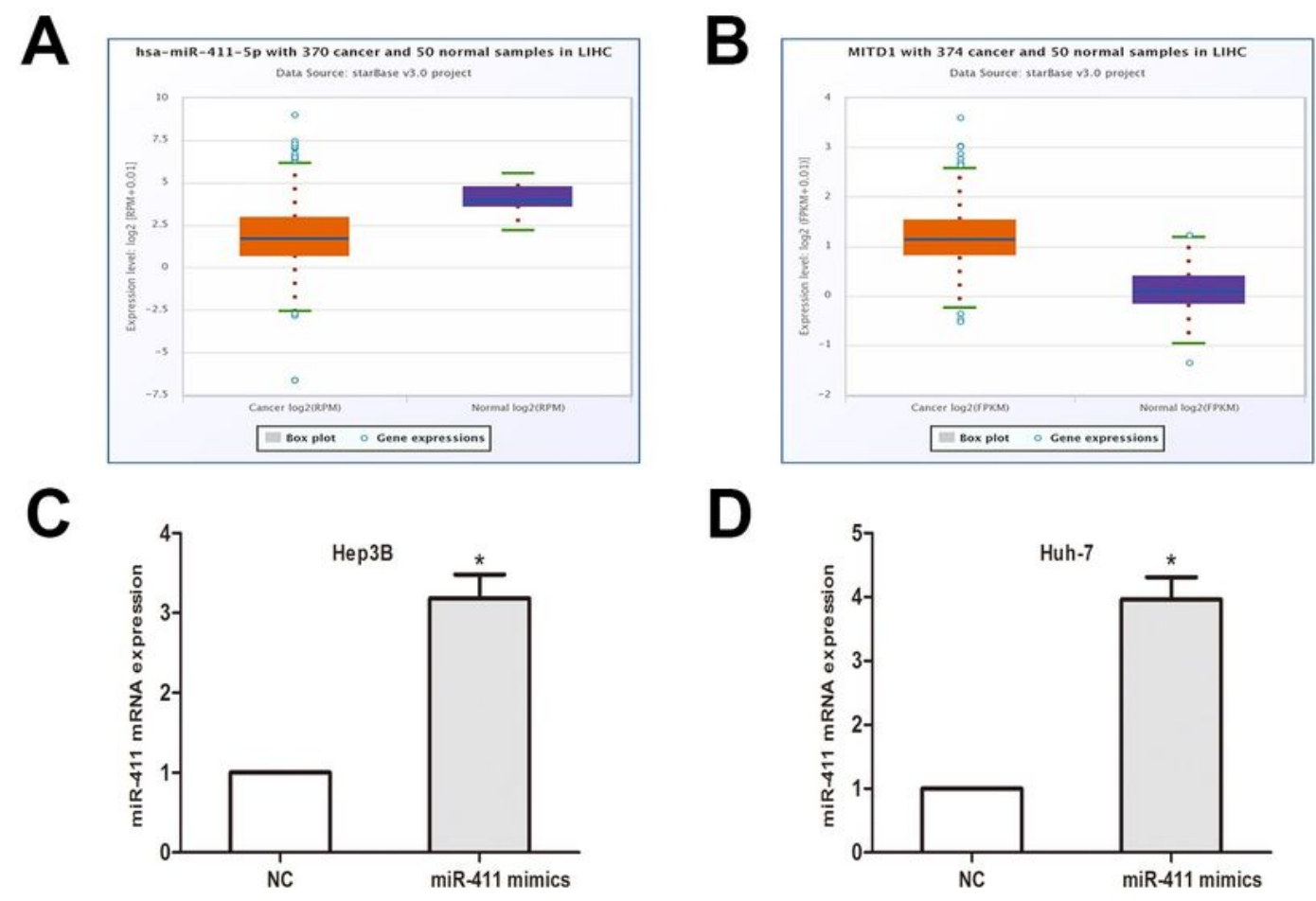

E

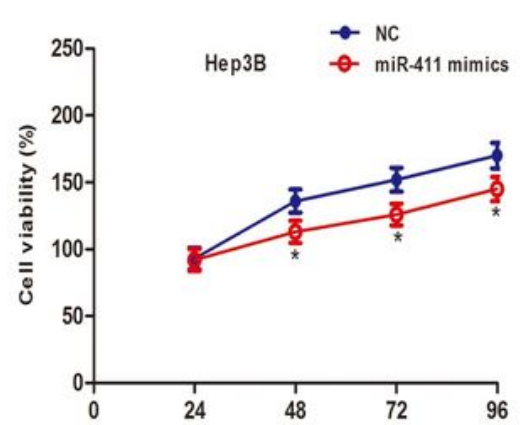

G

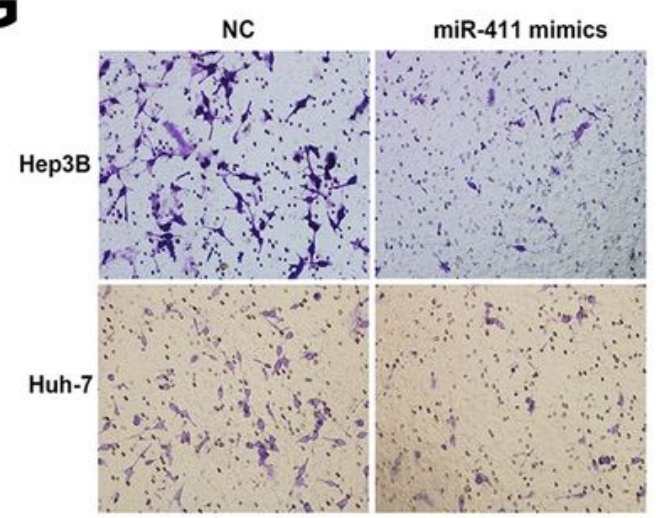

D

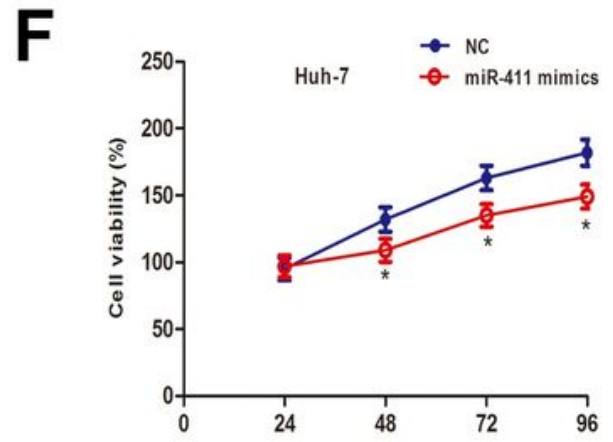

Figure 4

MiR-411 regulates malignant phenotypes of HCC cell. (A and B) Data from Starbase 3.0 showed the expression of miR-411 and MITD1 in HCC tissues and normal tissues. ( $C$ and D) qRT-PCR results showed miR-411 mimics were transfected into Hep3B and Huh-7 cells successfully. (E and F) CCK-8 assay was 
performed to detect cell viability of cells transfected with miR-411 mimics. (G) Transwell invasion assay was performed to investigate cell invasive ability. $\left({ }^{*} \mathrm{P}<0.05\right)$

A

Hsa-miR-411 3'GCAUGCGAUAUGCCAGAUGAU-5'

|| |||||

MIDT1 WT 5'-UAAUUUGUAUUAUGUCUACUU-3'

MIDT1 MUT $\quad$ 5'-UAAUUUGUAUUAUGCAGCUUU-3'
B

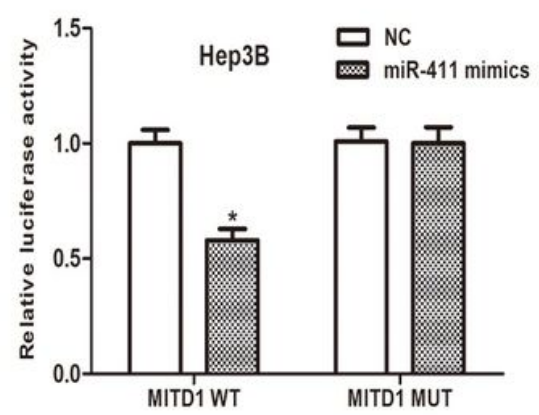

$\mathbf{E}$

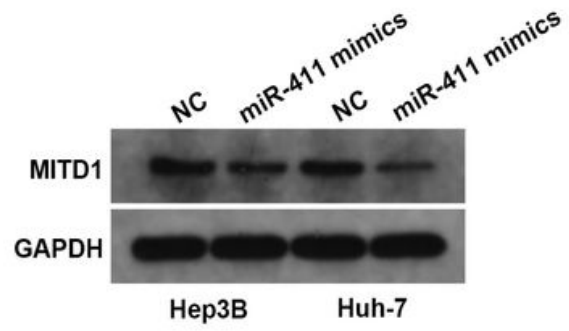

C

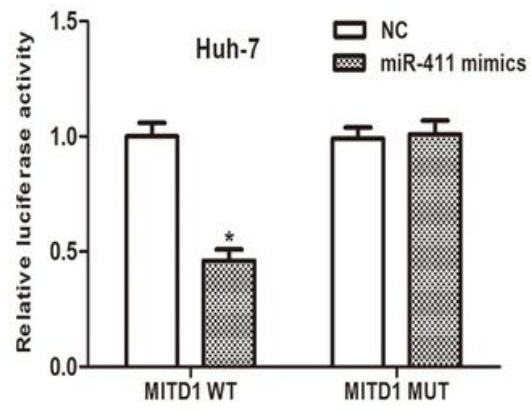

D

\section{Figure 5}

MiR-411 directly targets MITD1. (A) Sequence alignment of predicted miR-411 binding sites with the MITD1 3'UTR and the mutated sequence of miR-411. (B and C) Luciferase reporter assay was performed in HCC cells that were co-transfected with miR-411 mimics and reporter vectors that containing MITD1 3'UTR or mutated IGF1R 3'UTR. (D and E) Western blot analyses showed that overexpression of miR-411 markedly decrease the MITD1 expression level in HCC cells. ( $\left.{ }^{*}<0.05\right)$ 